\title{
Aspergillus Species Discrimination Using a Gas Sensor Array
}

\author{
Rosamaria Capuano ${ }^{1, *}$, Emilia Paba ${ }^{2}$, Antonella Mansi ${ }^{2}$, Anna Maria Marcelloni ${ }^{2}$, \\ Alessandra Chiominto ${ }^{2}$, Anna Rita Proietto ${ }^{2}$, Emiliano Zampetti ${ }^{3}{ }^{\circ}$, Antonella Macagnano ${ }^{3}{ }^{(1)}$,

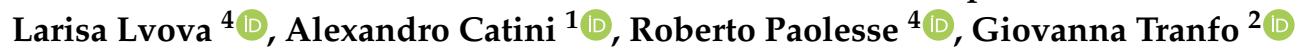 \\ and Corrado Di Natale ${ }^{1}$ \\ 1 Department of Electronic Engineering, University of Rome Tor Vergata, Via del Politecnico 1, \\ 00133 Rome, Italy; catini@ing.uniroma2.it (A.C.); dinatale@uniroma2.it (C.D.N.) \\ 2 Department of Occupational and Environmental Medicine, Epidemiology and Hygiene, National Institute \\ for Insurance against Accidents at Work (INAIL) Via Fontana Candida 1, \\ 00078 Monte Porzio Catone, Rome, Italy; e.paba@inail.it (E.P.); a.mansi@inail.it (A.M.); \\ a.marcelloni@inail.it (A.M.M.); a.chiominto@inail.it (A.C.); a.proietto@inail.it (A.R.P.); g.tranfo@inail.it (G.T.) \\ 3 Institute of Atmospheric Pollution Research-National Research Council, Via Salaria km. 29300, \\ 00016 Monterotondo, Rome, Italy; e.zampetti@iia.cnr.it (E.Z.); a.macagnano@iia.cnr.it (A.M.) \\ 4 Department of Chemical Science and Technology, University of Rome Tor Vergata, Via della Ricerca \\ Scientifica, 00133 Rome, Italy; larisa.lvova@uniroma2.it (L.L.); roberto.paolesse@uniroma2.it (R.P.) \\ * Correspondence: rmc.sensori@gmail.com; Tel.: +39-06-7259-7263
}

Received: 25 June 2020; Accepted: 15 July 2020; Published: 18 July 2020

\begin{abstract}
The efficiency of electronic noses in detecting and identifying microorganisms has been proven by several studies. Since volatile compounds change with the growth of colonies, the identification of strains is highly dependent on the growing conditions. In this paper, the effects of growth were investigated with different species of Aspergillus, which is one of the most studied microorganisms because of its implications in environmental and food safety. For this purpose, we used an electronic nose previously utilized for volatilome detection applications and based on eight porphyrins-functionalized quartz microbalances. The volatile organic compounds (VOCs) released by cultured fungi were measured at 3,5 , and 10 days after the incubation. The signals from the sensors showed that the pattern of VOCs evolve with time. In particular, the separation between the three studied strains progressively decreases with time. The three strains could still be identified despite the influence of culture time. Linear Discriminant Analysis (LDA) showed an overall accuracy of $88 \%$ and $71 \%$ in the training and test sets, respectively. These results indicate that the presence of microorganisms is detectable with respect to background, however, the difference between the strains changes with the incubation time.
\end{abstract}

Keywords: Aspergillus species; electronic nose; volatile organic compounds; porphyrins

\section{Introduction}

Fungal contamination of food and the environment may lead to the formation of products like mycotoxins and spores that can cause harmful effects to human health [1]. The fungi, Aspergillus has been the object of frequent studies. Aspergillus is a genus of filamentous fungi that contains around 250 species. A. flavus, A. fumigatus, and A. niger are the species most frequently involved in environmental contamination and associated with human diseases [2]. They are among the most abundant species in soil and for this reason they are also involved in disorders affecting plants and vegetable products [3,4]. In humans, A. fumigatus is an opportunistic pathogenic fungus that causes severe systemic infections and it is a major cause of fungal infections in immunocompromised 
patients [5]. It is classified in the second risk group according to its harmful properties (Italian Legislative Decree 81/2008). In addition to infectious diseases, A. fumigatus can induce allergic sensitization and symptomatic allergic lung disease [6] (invasive pulmonary aspergillosis, aspergilloma or different forms of hypersensitivity diseases), whose severity depends on the immunological status of the host.

With regard to the other species, both A. flavus and A. niger are of great interest in some occupational settings, especially in the agricultural and food sectors, as they are producers of mycotoxins, chemical toxic compounds that are produced via their secondary metabolism [7]. In suitable environmental conditions, A. flavus releases Aflatoxin B1 (AFB1), and it is the most toxic mycotoxin classified in Group 1 (carcinogenic to humans) by the International Agency for Research on Cancer (IARC). A. niger is a known producer of Ochratoxin $\mathrm{A}$; it is a potent nephrotoxin and has teratogenic, immunosuppressive and carcinogenic properties (Group 2B, IARC, Lyon, France).

The standard methods used to identify microbial species include culturing and biochemical tests, immunological assays, examining the antigen-antibody interaction, and polymerase chain reaction (PCR) for DNA identification. Although reliable, specific, and sensitive, these approaches are also expensive, time-consuming and labor-intensive [8,9]. Biosensor technology is expected to eliminate the drawbacks associated with biochemical tests, even though most biosensors are oriented to the detection of toxins rather than to the presence of microorganisms [10].

Alternative methods for the detection of fungi consider specific metabolic products and the volatile metabolites, known as the volatilome. The analysis of volatile compounds (VOCs) is attracting attention in many different fields, including clinical diagnosis, environmental monitoring, and food contamination [11].

Microbial volatile organic compounds are metabolic products of fungi, and bacteria and are formed in both primary and secondary metabolism as side-products. An on-line VOCs database (http://bioinformatics.charite.de/mvoc/index.php?site=home) reports more than 1000 volatile organic compounds originating from microorganisms; over 300 of them are classified as fungal VOCs [12]. These VOCs include alcohols, aldehydes, hydrocarbons, acids, ethers, esters, ketones, terpenes, furans, sulfur and nitrogen-containing compounds. The profile of the produced compounds is strictly correlated with microbial species and strains, incubation time and growth conditions such as substrate, nutrients, $\mathrm{pH}$, humidity and temperature [13].

Various analytical tools are suitable for detecting VOCs in different substrates. However, these techniques sometimes suffer from limitations that are typical of microbiological methods, that is, they often require sample pre-treatment, long-time analysis and complex data interpretation. Gas chromatography mass spectrometry is the typical standard for the analysis of gaseous compounds [14]. More advanced methods do not include the separation phase, and therefore, may have a fast analysis time [15]. These techniques have been applied to study Aspergillus volatilome in vitro and in real samples $[16,17]$.

In general, these studies indicate that different microorganisms produce very similar volatile compounds, but the differences between them are found in the global pattern of VOCs rather than in single compounds. It is important to note that, even if some VOC may be associated with a given species, the actual pattern released by the microorganisms may be variable because various factors can alter the species-dependent pattern. Among these factors, the substrate and the growing conditions are of paramount importance. Because of such variabilities, the patterns released by replicated cultures are affected by dispersion. Thus, pattern recognition is necessary to correctly attribute a measured pattern to a species [18].

Patterns of VOCs can be obtained with analytical instruments, measuring the abundance of individual compounds. Arrays of cross-selective sensors (electronic noses) are an alternative method to access the same information. These are ensembles of partially selective sensors that encode high-dimension patterns of VOCs into a smaller-dimension pattern of sensors signals. The encoding can preserve the relevant information necessary for the identification of samples. In such cases, 
electronic noses ensure the rapid and simple analysis of complex matrices of VOCs, as demonstrated in different fields such as medical diagnosis [19-21], the environment [22] and foods [23].

Several studies have reported the use of electronic noses for the identification and differentiation of microorganisms in vitro [24,25], and in real samples such as cereal grain [26,27] and yoghurt [28].

In this paper, the headspace of three species of Aspergillus were measured by an array of porphyrins-functionalized quartz crystal microbalance (QMB) sensors to correlate volatile organic compound patterns with the specific fungal species and the growth time. The results show that sensors can detect the presence of contamination but the identification of species may vary with the cultivation time.

\section{Materials and Methods}

\subsection{Samples}

Three American Type Culture Collection (ATCC) fungal strains belonging to Aspergillus genus (A. niger-9642, A. fumigatus-KM 8001, A. flavus-9643, LCG Standard) were considered.

Fungi were previously inoculated in Sabouraud Liquid Broth (SLB) and then isolated on Potato Dextrose Agar (PDA) plates and incubated at $25^{\circ} \mathrm{C}$ from 72 to $120 \mathrm{~h}$ in aerobic conditions.

In order to obtain cellular suspension at known concentrations, spores produced from different Aspergillus cultures were counted using a Burker counting chamber. Spore suspension from each Aspergillus species were plated onto replicated PDA petri dishes and incubated at $25^{\circ} \mathrm{C}$ for 10 days. Petri dishes containing the same quantity of PDA substrate were preserved during the experiment, and the headspace of these dishes were used as reference.

Experiments were carried out at the Biological Agents Risk Laboratory of the Department of Occupational and Environmental Medicine, Epidemiology and Hygiene, INAIL (Monteporzio Catone, Rome, Italy).

\subsection{The Electronic Nose}

Fungi headspaces were measured using a gas sensor array designed and developed at the University of Rome Tor Vergata.

The array was made of eight quartz microbalance (QMB) sensors. In this device, mass changes $(\Delta \mathrm{m})$ on the quartz surface result in a frequency shift $(\Delta \mathrm{f})$ in the electrical output signal of the oscillator circuit. In the theory of small perturbations, $\Delta \mathrm{f}$ is linearly proportional to $\Delta \mathrm{m}$ [29]. The adopted QMBs were AT-cut quartz, with a fundamental frequency of $20 \mathrm{MHz}$, corresponding to a mass resolution of the order of a few nanograms/Hz.

The QMBs were functionalized by seven metal complexes ( $\mathrm{Mg}, \mathrm{Co}, \mathrm{Cu}, \mathrm{Zn}, \mathrm{Fe}, \mathrm{Mn}, \mathrm{Sn})$ and a free base $\left(\mathrm{H}_{2}\right)$ of 5, 10, 15, 20-tetrakis-(4-butyloxyphenyl) porphyrin (TBPP) [30]. Porphyrin films were deposited on the quartz surface by spray casting.

Each QMB is connected to an oscillator circuit. The frequency of the output signals of the oscillators is sequentially measured using a reference clock provided by a temperature compensated quartz that achieves a frequency resolution equal to $0.1 \mathrm{~Hz}$. The gas sensor array is complemented by temperature and relative humidity sensors. The sample is delivered through an embedded tubeless pneumatic system manifold. Input inlets are connected to a miniature diaphragm pump, a three-way valve, a proportional valve and a mass flow sensor. The sample flow was $50 \mathrm{sccm}$.

The instrument is connected and powered via a USB. In-house software in Matlab controls the instrument and the data acquisition.

Sensors were calibrated by measuring their response to a series of compounds that are representative of different chemical families, including cyclohexane, hexane, ethanol, xylene, styrene, hexanal, benzaldehyde, acetone, triethylamine, and acetic acid. The vapors of the volatile compounds were generated by bubbling a nitrogen gas stream into the liquid compound and diluting the vapor with nitrogen gas. The dilution rate was controlled by computer-driven mass-flow controllers (MKS). 
The concentration of the volatile compounds was calculated by Antoine's law using the parameters listed in the NIST database (http://webbook.nist.gov/chemistry).

\subsection{Headspace Analysis}

Petri dishes containing Aspergillus culture were closed with an aluminum lid specifically designed for this experiment. The lid was shaped in order to seal the fungi culture from the surrounding environment to allow the formation of a headspace. About five minutes was necessary to establish a stable headspace composition. The volatile organic compounds released by Aspergillus were sampled and analyzed for one minute at a flow-rate of $50 \mathrm{sccm}$ using the pump embedded in the gas sensor array. A filter with a porosity of $0.8 \mu \mathrm{m}$ (Sartorius, Germany) was used during the analysis in order to avoid the migration of spores from the samples to the device. The reference samples required for a stable sensors baseline was obtained by flowing the laboratory air through a $\mathrm{CaCl}_{2}$ bed. Measurements were carried out under a biohazard hood.

Figure 1 shows the experimental set up. The cultures were analyzed 3, 5, and 10 days after the first inoculation.

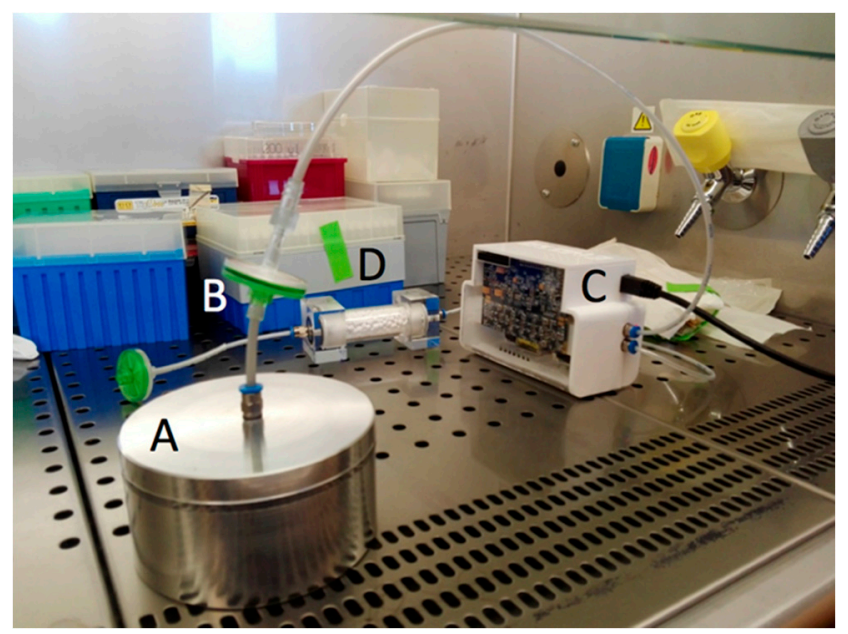

Figure 1. Picture showing the experimental system set-up under a biohazard hood. (A) Petri dish covered with customized lid suitable to ensure a stable headspace; (B) filter used to block spore migration from Aspergillus culture to sensors; (C) the electronic nose; (D) the $\mathrm{CaCl}_{2}$ trap.

\subsection{Data Analysis}

The sensor response consists of the variation in the resonant frequency measured at the beginning and at the end of the exposure to the sample. Each measurement results in an eight-dimensional vector, which is comprised of the responses of the individual sensors.

Data were analyzed using Principal Component Analysis (PCA) [31] to study the clustering of the sensors' data with respect to the different species of fungi and to the temporal evolution of the VOCs. Linear Discriminant Analysis (LDA) [32] was used as a classifier to estimate the discrimination capabilities of the sensor array. The statistical significance of the sensor signals, and PCA scores were evaluated with the non-parametric Kruskal-Wallis rank sum test followed by Bonferroni correction in case of multiple comparisons.

All data analysis was performed in Matlab ${ }^{\circledR}$ R2020a using Statistics and Machine Learning toolboxes.

\section{Results and Discussion}

Before we investigated the headspace of the microorganisms, the sensor array was characterized with respect to a set of generic volatile compounds. For this purpose, sensors were exposed to various concentrations of ten volatile compounds, which were chosen because they were most representative 
of different families. The concentration of the compounds was modulated by changing the dilution of the saturation pressure in a stream of nitrogen gas.

The sensors' data were normalized by dividing the response of each sensor by the sum of the responses of all the sensors in the array [33]. The effects of different concentrations are largely removed in the normalized data and the VOCs can be identified without the confounding influence of variable concentrations [34].

Figure 2 shows the results of the PCA of the normalized characterization data. Except for the partial overlap of styrene and xylene, the data for each VOC form close clusters that are well separated from the others. The arrangement of VOCs in the scores plot is consistent with the chemical families. The sensors are uniformly distributed in the bi-plot. For instance, Mn-TBPP and Fe-TBPP make a large contribution with respect to alkanes (cyclohexane and hexane), while Co-TBPP is dominant with respect to polar compounds (ethanol and acetic acid).
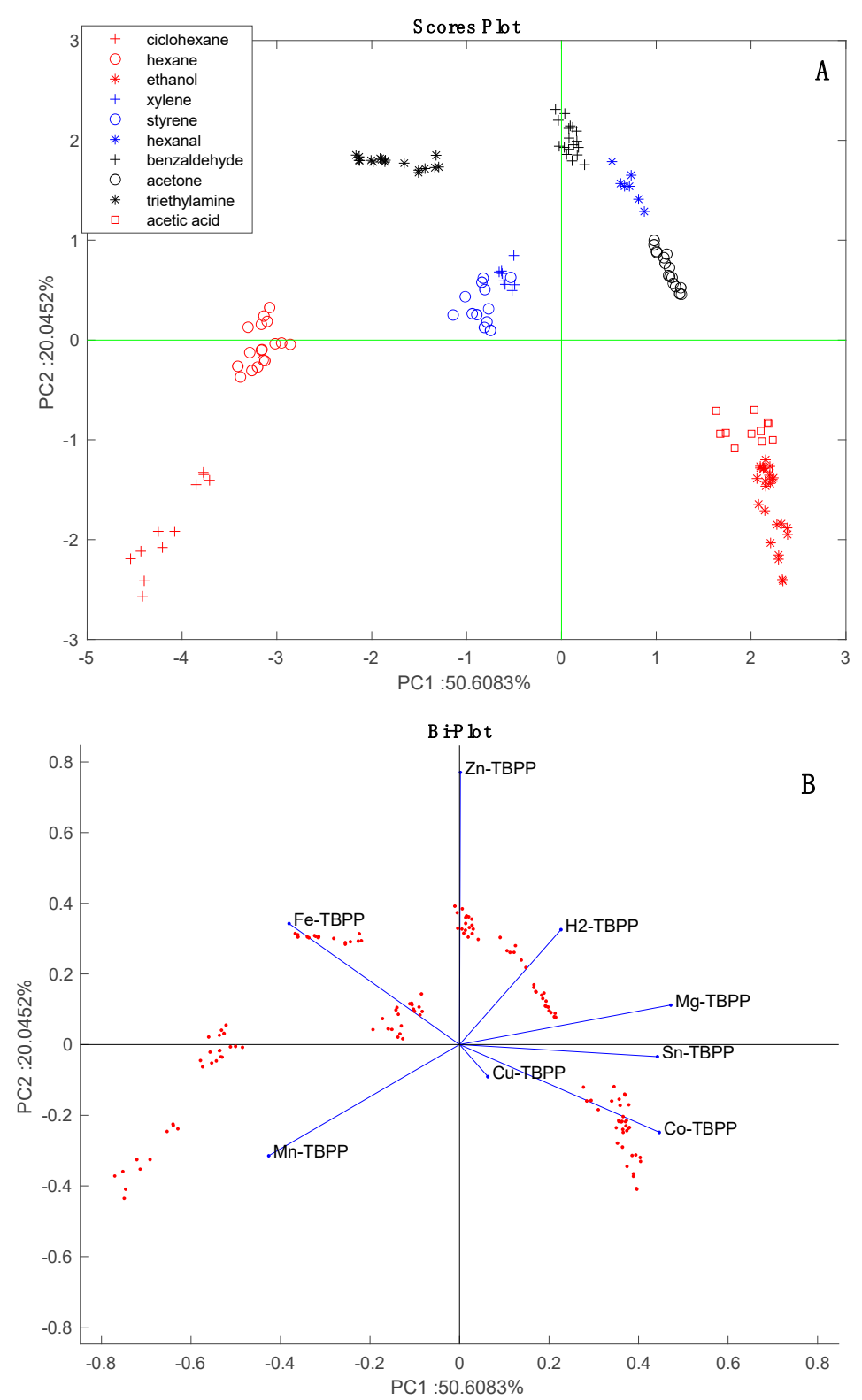

Figure 2. Principal Component Analysis (PCA) of characterization data. (A) Scores plot, (B) bi-plot which shows the loadings of the sensors. 
The analysis of Aspergillus spp. headspace was carried out in replicated cultures in order to examine the biological variability in independent cultures.

Figure 3 shows the sequence of the signals recorded by one of the sensors (Zn-TBPP) during three consecutive measurements of the headspace of cultures of A. niger, A. flavus and A. fumigatus. After each exposure to culture VOCs, the signal returns to its original baseline. The reversibility of the sensors is not absolute, however, such a limited hysteresis does not affect the result of the analysis.

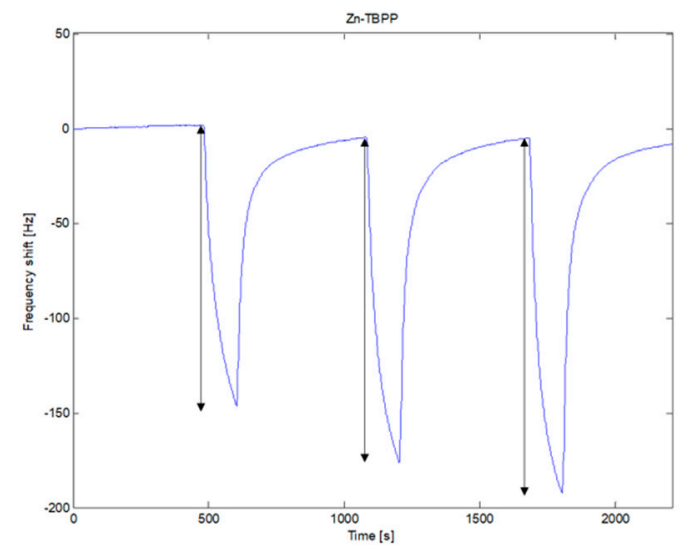

Figure 3. Signal recorded by the Zn-TBPP sensor. It shows the sensor behavior for the sequence of exposures to the headspace of A. niger, A. flavus and A. fumigatus in order. Arrows indicates the frequency shift considered as the sensor response and used in the data analysis. The response time is $135 \mathrm{~s}$.

The absorption of VOCs increases the mass on the QMB, then the frequency shift is negative. However, it is usual to consider the absolute value of the difference of the frequency before and at the end of the exposure as the sensor response. This value was used for all the following analysis.

Figure 4 shows the response of the sensors to the headspace of fungi compared to the response to blank samples (PDA) measured after 3, 5 and 10 days. Blank PDA dishes were not inoculated with Aspergillus, however, they were left exposed to the laboratory air. Environmental contamination was not tested but it could not be excluded.
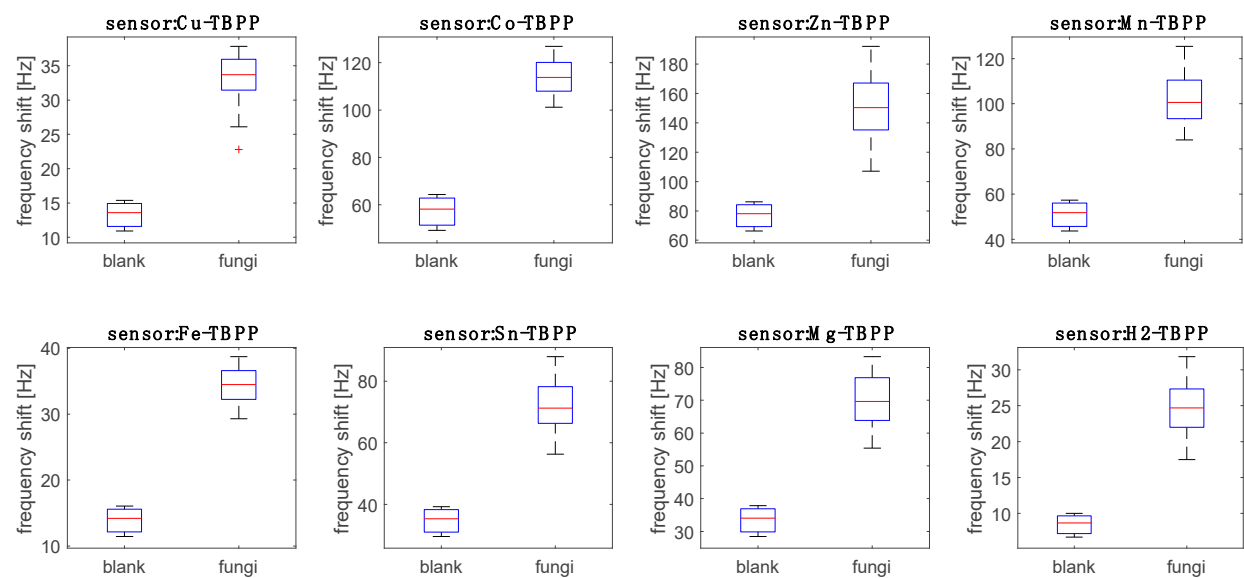

Figure 4. Distribution of the sensor responses to uncontaminated culture media and media inoculated with fungi. Each group contains data obtained at days 3, 5, and 10 after the first inoculation. The distribution of the responses is represented by a box-plot calculated with the Matlab embedded function. In each box, the central mark is the median, the edges are the 25 th and 75 th percentiles, the whiskers extend to the most extreme data points not considered outliers. Outliers, labeled with a cross, are plotted individually. 
All sensors' signals show a large and statistically meaningful difference between fungi and PDA. Thus, the VOCs released by blank culture media during the experiment did not have an effect on the sensors' response to fungi.

The sensors' data for the Aspergillus headspace were sorted into nine groups corresponding to the three strains and to the three inoculation times (days 3, 5, and 10). Figure 4 shows the distribution of the responses of the sensors with respect to the nine groups.

Figure 5 shows that both strain and time of culture influence the response of the sensors. The sensors demonstrate similar behavior, and in this regard, it is important to note that most of the correlation between the sensors' data is due to the abundance of VOCs in the headspace. The relative response to the three strains changes with the time since inoculation.
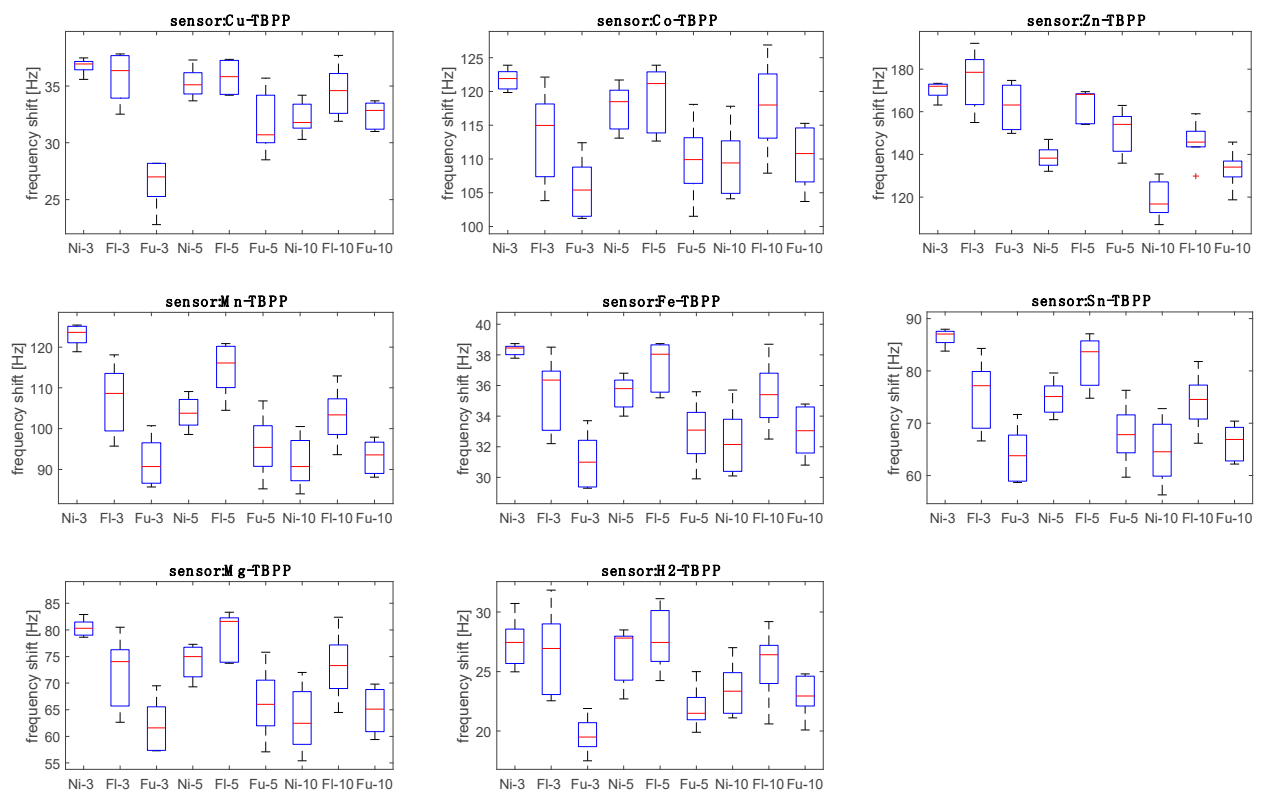

Figure 5. The distributions of the responses of sensors in the nine groups are represented by box-plots. Groups are labeled with the initials of the strain $(\mathrm{Ni}, \mathrm{Flu}$, and $\mathrm{Fu})$ and a number $(3,5,10)$ indicating the days since inoculation. For a description of the box-plot details, see the caption of Figure 4.

This suggests that the real-time evolution of the volatilome composition is nonlinear. Previous studies suggest that the relationship between the abundance of volatile compounds and the time from inoculation shows particular trends for each compound [35]. For instance, in Aspergillus fumigatus, the abundance of some ketones (2-pentanone, 2-heptanone, 2-octanone and 2-nonanone) strongly decreased after three days while the changes in alcohol content was observed after seven days. Different behaviors were observed for alcohols. For example, from day 10 to day 14, the abundance of 3-Methyl-1-butanol decreased in Aspergillus fumigatus but increased in Aspergillus niger [36].

Before further analyzing the data in order to determine the differences between strains it is interesting to compare the responses to Aspergillus species with responses to the pure VOCs. The data regarding the headspace of the microorganism headspace was projected onto the scores plot shown in Figure 2. Figure 6 shows that with respect to pure VOCs, Aspergillus data form a compact cluster lying between alcohols, aromatics, and alkanes. This result is consistent with the fact that alcohols (2-methyl-1-propanol and 2-ethyl-hexanol), ketones (2,4,pentanedione and 2-heptanone) and aromatics (3-methylfurane) are among the most frequently identified components of Aspergillus volatilome [16].

A better appraisal of the sensors' data can be achieved by considering the multivariate analysis of the sensor responses. Hence, PCA and LDA were applied to the data.

PCA is an unsupervised algorithm that is useful to study the correlations between data and the correlations between sensors. On the other hand, LDA, being supervised, provides an estimate of the ability of the sensor array to identify the various groups. 


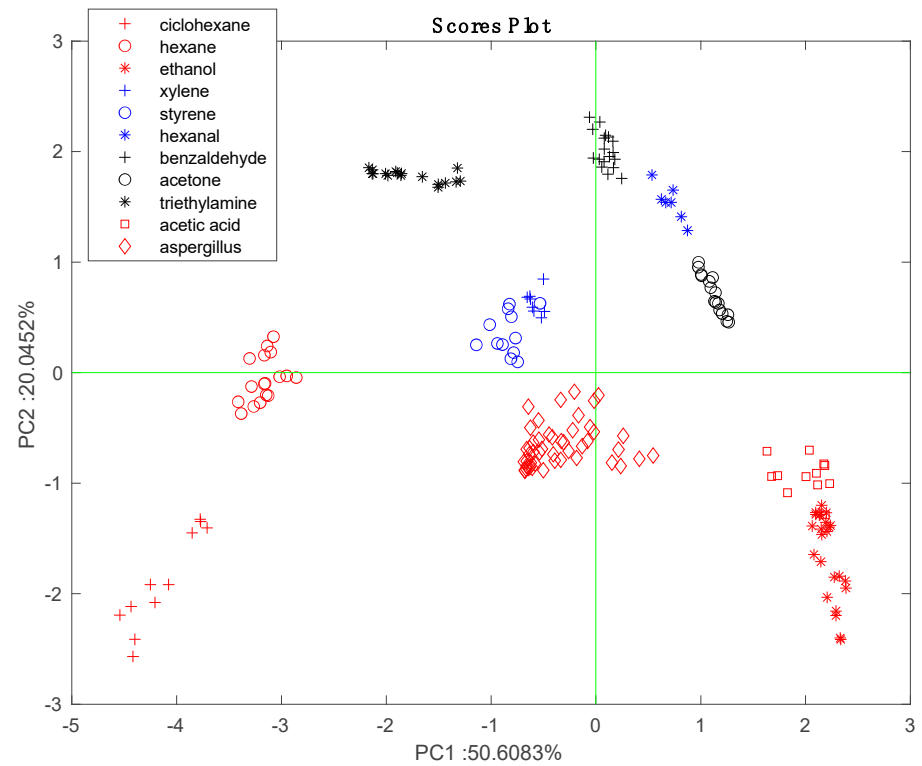

Figure 6. Sensors' data related to Aspergillus headspaces were normalized and projected onto the scores plot of the PCA calculated with the characterization data and shown in Figure 2. Microorganisms' data form a close cluster between aromatics and polar compounds.

Before calculating the PCA, the data was normalized by dividing the sensor signals by the sum of signals of all sensors.

Analysis of variance (non-parametric Kruskal-Wallis rank sum) was applied to the principal components in order to select those that achieved the largest separation of the Aspergillus species. Figure 7 shows the corresponding $p$-value calculated for the first four principal components that explain more than $1 \%$ of the total variance. The first two principal components (which explain $81.5 \%$ and $14.5 \%$ of the total variance, respectively) show the largest separation among the classes.

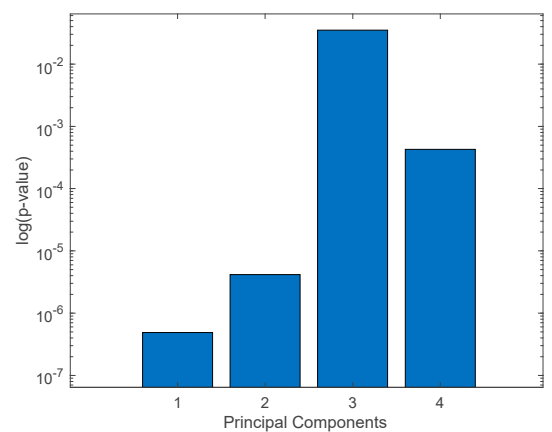

Figure 7. Plot of the logarithm of the probability of null hypothesis ( $p$-value) returned by a non-parametric Kruskal-Wallis rank sum. The $p$-value is related to the largest separation between at least two of the nine groups.

The scores in the plane of PC1 and PC2 are plotted in Figure 8. This plot shows the two influences on the data: strain and incubation time. The effect of the incubation time is observed along PC1 while the separation between the strains is observed along PC2. Since the variance explained by PC1 is more than five times larger than that explained by PC2, we can conclude that the largest variability in the data is due to the change in volatilome occurring as the cultures grow. It is important to note that time has no influence on the data from the blank samples, thus, the effect cannot be merely due to sensors drift.

The loading of the sensors to the PCA scores plot are shown in Figure 8B. With respect to PC1, all sensors are oriented towards the increment of time except $\mathrm{Zn}$-TBPP. With respect to PC2, Co-TBPP 
singularly points to A. fumigatus samples. In Figure 2B, these sensors contribute most to aldehydes and amines (Zn-TBPP) and polar compounds (Co-TBPP). Thus, based on his behavior we can surmise a prevalence of polar compounds in A. fumigatus and a decrease in aldehydes along the incubation time. Further investigation is necessary to confirm this conjecture.

Although, incubation time is the dominant source of variability in the data, we also wanted to investigate if the data contain also enough information to identify the strains regardless of their incubation time. Therefore, LDA was used to calculate a classifier for the recognition of three strains, A. niger, A. flavus, and A. fumigatus.

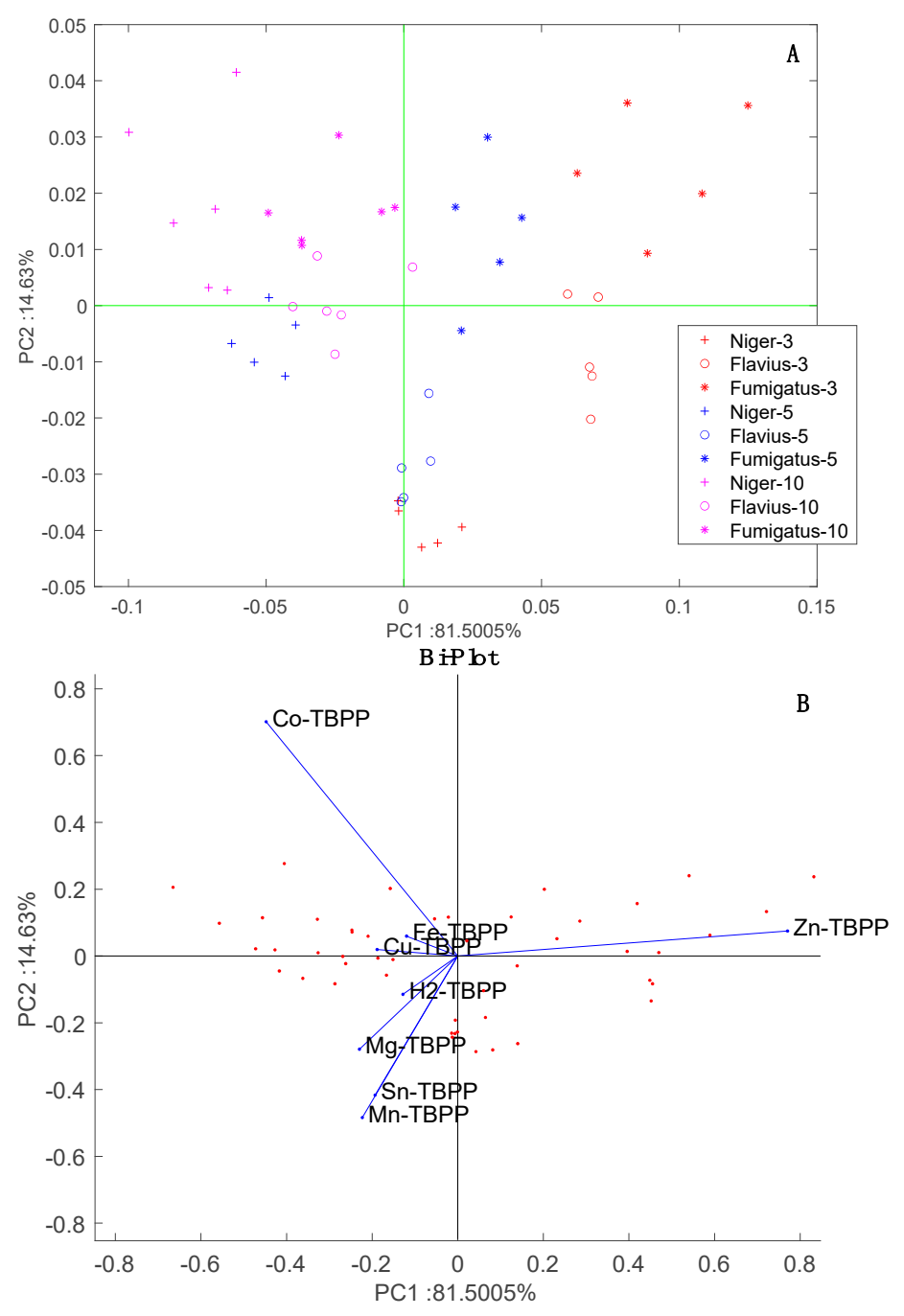

Figure 8. PCA of Aspergillus headspace data. (A) scores plot, (B) bi-plot where the loadings of sensors are indicated. In Figure 8A, Aspergillus species are marked with the same symbol whose colors identifies the time after incubation.

Due to the limited sample size, the classification performance is strongly dependent on how the data are split in the training and test sets. To avoid misinterpretation of the data, the whole dataset was randomly split into training and test sets and the LDA was calculated for each split. Figure 9 shows the accuracy in the training and test sets for each random split. The accuracies distribution is well fitted by a bivariate gaussian. In Figure 9A, the mean and the ellipsoid associated to the covariance matrix are shown while in Figure 9B the occurrence of a couple of accuracies in training and test are shown. 


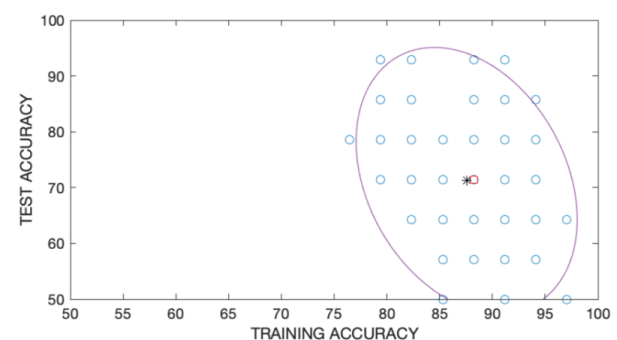

(A)

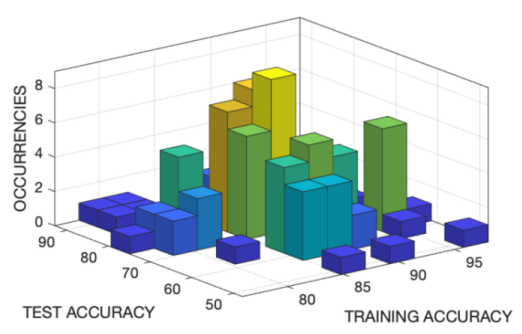

(B)

Figure 9. (A) Plot of accuracy of the Linear Discriminant Analysis (LDA) classifier for 100 random partitions of the data in training and test sets. (B) Frequency of occurrence of the multivariate variable made by the accuracy in training and test sets. The ellipse in Figure 9A shows the covariance matrix of the bi-variate normal distribution. The mean value, corresponding to the center of the covariance matrix, was chosen to represent the results.

Table 1 shows the confusion matrix for the mean of the distribution of accuracy in the training and test sets. In total, about $88.2 \%$ of the samples were correctly identified in training and $71.4 \%$ in test.

Thus, even if the variability due to the different strains is residual with respect to the incubation time, it enables the identification of the species of Aspergillus.

Table 1. Confusion matrix of the LDA model in training and test. Correct identifications are emphasized with bold font.

\begin{tabular}{cccc}
\hline & \multicolumn{4}{c}{ Training } \\
\cline { 2 - 4 } & Asp. Niger & Asp. Flavius & Asp. Fumigatus \\
\hline Asp. Niger & $\mathbf{1 1}$ & 1 & 1 \\
\hline Asp. Flavius & 1 & $\mathbf{8}$ & 0 \\
\hline Asp Fumigatus & 0 & 1 & $\mathbf{1 1}$ \\
\hline & & Test & Asp. Fumigatus \\
\cline { 2 - 4 } & Asp. Niger & Asp. Flavius & 0 \\
\hline Asp. Niger & $\mathbf{4}$ & 1 & 0 \\
\hline Asp. Flavius & 0 & $\mathbf{2}$ & $\mathbf{4}$ \\
\hline Asp Fumigatus & 0 & 3 &
\end{tabular}

\section{Conclusions}

This paper reports the application of a gas sensor array to in vitro discrimination of $A$. niger, A. fumigatus and A. flavus species, which are often involved in environmental and food contamination. Previous studies, carried out with analytical instrumentation, have shown that each species is characterized by a unique pattern of VOCs. However, the abundance of each VOC and its evolution along the cultivation time is variable and it depends on the species, as well as the culture media.

Sensors detect the presence of the fungi with respect to the blank sample, but the difference between the species depends on the cultivation time. This finding is corroborated by previous investigations that show that the abundance of each VOC has a proper rate of evolution with the cultivation time. It is particularly interesting that very similar compounds, for which sensors are expected to have the same sensitivity, may decrease in Aspergillus fumigatus but increase in Aspergillus niger [33]. This behavior suggests the possibility of detecting microorganisms independently from the growth conditions (time and culture media). Our data show that the processes occurring along the incubation time contributes significantly to the variance in the data; however, even though it is not predominant, the information related to the strain is captured by the sensors and can be used to identify the strains independently of the incubation conditions. 
In this study the performance of the sensors was not considered to be optimal for practical routine applications. On the other hand, the composition of the sensor array was not optimized for this purpose. A detailed investigation of the chemistry of Aspergillus genus volatilome could be used to make a careful choice of sensitive materials to design electronic noses that could be used for rapid and non-invasive detection of fungal contamination.

Such studies will require a wider experimental design that includes growth in different media and incubation parameters (e.g., temperature and humidity). Furthermore, although the chosen species are among the most interesting in the Aspergillus genus, an extension of the study to other members of the genus should be considered for a thorough appraisal of the ability of electronic noses to identify similar microorganisms.

Author Contributions: Conceptualization and design of experiment, C.D.N., E.P., A.M. (Antonella Mansi), and G.T.; synthesis of molecules and sensor preparation, R.P., R.C., A.M. (Antonella Macagnano) and E.Z.; performed the experiment, R.C., A.C. (Alexandro Catini), A.M. (Antonella Mansi), A.M.M., A.C. (Alessandra Chiominto), A.R.P., L.L.; data analysis, C.D.N., R.C.; and writing, C.D.N., R.C. All authors have read and agreed to the published version of the manuscript.

Funding: This study was funded by INAIL within the framework of the project BRIC 2016-ID12.

Conflicts of Interest: The authors declare no conflict of interest.

\section{References}

1. Wen, J.; Mu, P.; Deng, Y. Mycotoxins: Cytotoxicity and biotransformation in animal cells. Toxicol. Res. 2016, 5, 377. [CrossRef] [PubMed]

2. Russell, R.; Paterson, M.; Lima, N. Filamentous fungal human pathogens from food emphasizing Aspergillus, Fusarium and Mucor. Microorganisms 2017, 5, 44.

3. Klich, M.A. Health effects of Aspergillus in food and air. Toxicol. Ind. Health 2009, 25, 657-667. [CrossRef]

4. Perrone, G.; Susca, A.; Cozzi, G.; Ehrlich, K.; Varga, J.; Frisvad, J.C.; Meijer, M.; Noonim, P.; Mahakarnchanakul, W.; Samson, R.A. Biodiversity of Aspergillus species in some important agricultural products. Stud. Mycol. 2007, 59, 53-66. [CrossRef]

5. Behnsen, J.; Hartmannm, A.; Schmaler, J.; Gehrke, A.; Brakhage, A.; Zipfel, P. The Opportunistic Human Pathogenic Fungus Aspergillus fumigatus Evades the Host Complement System. Infect. Immun. 2008, 76, 820-827. [CrossRef]

6. Sugui, J.; Kwon-Chung, K.; Juvvadi, P.; LAtge, J.; Steinbach, W. Apsergillus fumigatus and related species. Cold Spring Harb. Perspect Med. 2014, 5, a019786. [CrossRef] [PubMed]

7. Varga, J.; Baranyi, N.; Chandrasekaran, M.; Vagvolgyi, C.; Kocsube, S. Mycotoxin producers in Aspergillus genus: An update. Acta Biol. Szeged. 2015, 59, 151-167.

8. Varga, J.; Juhász, Á.; Kevei, F.; Kozakiewicz, Z. Molecular diversity of agriculturally important Aspergillus species. Eur. J. Plant Pathol. 2004, 110, 627-640. [CrossRef]

9. Kozakiewicz, Z. Aspergillus Species on Stored Products. Myc. Papers 1989, 161, 1-188.

10. Wang, X.; Niessner, R.; Tang, D.; Knopp, W. Nanoparticle based immunosensors and immunoassays for aflatoxins. Anal. Chim. Acta 2016, 912, 10-23. [CrossRef]

11. Scott-Thomas, A.; Chambers, T.S. Volatile Organic Compounds: Upcoming Role in Diagnosis of Invasive Mould Infections. Curr. Fungal Infect. Rep. 2017, 11, 117-123. [CrossRef]

12. Lemfack, M.C.; Gohlke, B.; Preissner, S.; Piechulla, B.; Preissner, R. mVOC 2.0: A database of microbial volatiles. Nucleic Acids Res. 2017, 46, D1261-D1265. [CrossRef] [PubMed]

13. Korpi, A.; Jarnberg, J.; Pasanen, A.-L. Microbial volatile organic compounds. Crit. Rev. Toxicol. 2009, 39, 139-193. [CrossRef] [PubMed]

14. Wang, Y.; Li, Y.; Yang, J.; Ruan, J.; Sun, C. Microbial volatile organic compounds and their application in microorganism identification in foodstuff. TrAC Trend. Anal Chem. 2016, 78, 1-16. [CrossRef]

15. Zhu, J.; Hill, J.E. Detection of Escherichia coli via VOC profiling using secondary electrospray ionization-mass spectrometry (SESI-MS). Food Microbiol. 2013, 34, 412-417. [CrossRef] [PubMed] 
16. Pennerman, K.K.; Al-Maliki, H.S.; Lee, S.; Bennett, J.W. Fungal volatile organic compounds (VOCs) and the genus Aspergillus. In New and Future Developments in Microbial Biotechnology and Bioengineering; Elsevier: Amsterdam, The Netherlands, 2016; pp. 95-115.

17. Vinaixa, M.; Marín, S.; Brezmes, J.; Llobet, E.; Vilanova, X.; Correig, X.; Ramos, A.; Sanchis, V. Early detection of fungal growth in bakery products by use of an electronic nose based on mass spectrometry. J. Agric. Food Chem. 2004, 52, 6068-6074. [CrossRef]

18. Van der Greef, J.; Smilde, A. Symbiosis of chemometrics and metabolomics past, present, and future. J. Chemom. 2005, 19, 376-386. [CrossRef]

19. Di Natale, C.; Paolesse, R.; Martinelli, E.; Capuano, R. Solid-state gas sensors for breath analysis: A review. Anal. Chim. Acta. 2014, 824, 1-17. [CrossRef]

20. Jalal, A.; Alam, F.; Roychoudury, S.; Umasnakar, Y.; Pala, N.; Bhansali, S. Prospects and Challenges of Volatile Organic Compound Sensors in Human Healthcare. ACS Sens. 2018, 3, 1246-1263. [CrossRef]

21. Krilaviciute, A.; Heiss, J.A.; Leja, M.; Kupcinskas, J.; Haick, H.; Brenner, H. Detection of cancer through exhaled breath: A systematic review. Oncotarget 2015, 6, 38643-38657. [CrossRef]

22. Capelli, L.; Sironi, S.; Del Rosso, R. Electronic Noses for Environmental Monitoring Applications. Sensors 2014, 14, 19979-20007. [CrossRef] [PubMed]

23. Loutfi, A.; Coradeschi, S.; Mani, G.K.; Shankar, P.; Rayappan, J.B.B. Electronic noses for food quality: A review. J. Food Eng. 2015, 144, 103-111. [CrossRef]

24. Zhang, Y.; Asakim, J.; Zhong, W.; Orlean, P.; Suslick, K. Identification of pathogenic fungi with an optoelectronic nose. Analyst 2014, 139, 1922-1928. [CrossRef] [PubMed]

25. Zetola, N.; Modongo, C.; Matlhagela, K.; Sepako, E.; Matsiri, O.; Tamuhla, T.; Mbongwe, B.; Martinelli, E.; Sirugo, G.; Di Natale, C. Identification of a large pool of microorganisms with an array of porphyrin based gas sensors. Sensors 2016, 16, 466. [CrossRef] [PubMed]

26. Eifler, J.; Martinelli, E.; Santonico, M.; Capuano, R.; Schild, D.; Di Natale, C. Differential detection of potentially hazardous Fusarium species in wheat grains by an electronic nose. PLoS ONE 2011, 6, e21026. [CrossRef] [PubMed]

27. Jiarpinijun, A.; Osako, K.; Siripatrawan, U. Visualization of volatomic profiles for early detection of fungal infection on storage Jasmine brown rice using electronic nose coupled with chemometrics. Measurement 2020, 157, 107561. [CrossRef]

28. Ndagijimana, M.; Chaves-Lopez, C.; Corsetti, A.; Tofalo, R.; Sergi, M.M.; Paparella, A.; Guerzoni, M.E.; Suzzi, G. Growth and metabolites production by Penicillium brevicompactum in yoghurt. Int. J. Food Microbiol. 2008, 127, 276-283. [CrossRef]

29. Magna, G.; Belugina, R.; Mandoj, F.; Catini, A.; Legin, A.; Paolesse, R.; Di Natale, C. Experimental determination of the mass sensitivity of quartz microbalances coated by an optical dye. Sens. Actuators $B$ Chem. 2020, 320, 128373. [CrossRef]

30. Paolesse, R.; Nardis, S.; Monti, D.; Stefanelli, M.; Di Natale, C. Porphyrinoids for Chemical Sensor Applications. Chem. Rev. 2017, 117, 2517-2583. [CrossRef]

31. Wold, S.; Esbensen, K.; Geladi, P. Principal component analysis. Chemom. Intell. Lab. Syst. 1987, 2, 37-52. [CrossRef]

32. Tharwat, A.; Gaber, T.; Ibrahim, A.; Hassanien, A. Linear discriminant analysis a detailed tutorial. AI Commun. 2017, 30, 169-190. [CrossRef]

33. Horner, G.; Hierold, C. Gas analysis by partial model building. Sens. Actuators B Chem. 1990, 3, 173-184. [CrossRef]

34. Catini, A.; Kumar, R.; Capuano, R.; Martinelli, E.; Paolesse, R.; Di Natale, C. An exploration of the metal dependent selectivity of a metalloporphyrins coated quartz microbalances array. Sensors 2016, 16, 1640. [CrossRef] [PubMed]

35. Gao, P.; Korley, F.; Martin, J.; Chen, B. Determination of unique microbial volatile organic compounds produced by five Aspergillus species commonly found in problem buildings. AIHA J. 2002, 2, 135-140. [CrossRef]

36. Matysik, S.; Herbarth, O.; Mueller, A. Determination of volatile metabolites originating from mould growth on wall and synthetic media. J. Microbiol. Methods 2008, 75, 182-187. [CrossRef]

(C) 2020 by the authors. Licensee MDPI, Basel, Switzerland. This article is an open access article distributed under the terms and conditions of the Creative Commons Attribution (CC BY) license (http://creativecommons.org/licenses/by/4.0/). 